\title{
Evaluation of Clinical Innovation: A Gray Zone in the Ethics of Modern Clinical Practice?
}

\author{
Johane Patenaude, $P h D^{7}$, Andrew M. Grant, MB, ChB, MRCP, FRCPC, FACMI, DPhil', \\ Marianne Xhignesse, MD, MSc ${ }^{3}$, Frédéric Leblanc, $M S c^{4}$, and Josiane Courteau, $P h D^{5}$
}

'Department of Surgery, Faculty of Medicine and Health Sciences, Université de Sherbrooke, Sherbrooke, QC, Canada; ${ }^{2}$ Collaborative Research for Effective Diagnostics, Department of Biochemistry, Faculty of Medicine and Health Sciences, Université de Sherbrooke, Sherbrooke, QC, Canada; ${ }^{3}$ Department of Family Medicine, Chaire Lucie et André Chagnon pour l'enseignement d'une approche intégrée

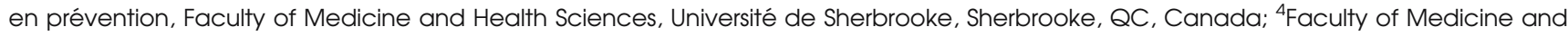
Health Sciences, Université de Sherbrooke, Sherbrooke, QC, Canada; ${ }^{5}$ Research Clinical Center, Centre hospitalier universitaire de Sherbrooke, Sherbrooke, QC, Canada.

BACKGROUND: Various stakeholders can have differing opinions regarding ethical review when introducing new procedures with patients.

OBJECTIVE: This pilot study examines the way in which Research Ethics Boards (REBs; Institutional Review Boards) and clinical biochemists (CBs; laboratory medicine specialists) differ in their interpretation of what is research and what should be considered common practice versus innovation versus experimentation when introducing new procedures with patients. It also explores whether these groups agree on who is responsible for the ethical review of new procedures.

METHODS: A validated case scenario for the introduction of a new diagnostic test into clinical practice was sent to CBs and REBs across Canada. Participants were asked to determine whether the scenario constituted research; whether the test procedure should be considered as experimental, innovative, or commonly accepted care; and whether the project required approval by a REB and, if not, who should be responsible for ethical review.

RESULTS: Results showed $81 \%$ of $37 \mathrm{CBs}$ and $52 \%$ of 27 REBs identified the scenario as research. Responsibility for ethical review was assigned to REBs by $44 \%$ of REBs and $54 \%$ of CBs. Of all participants, 53\% classified the test procedure as 'innovative', 8\% as 'experimental', whereas $17 \%$ classified it as 'commonly accepted'.

CONCLUSIONS: This pilot study indicates a substantial variation in the ethical assessment of innovation in clinical care. This suggests the need to further elaborate on the types of innovation in health care and categorize the nature of the risks associated with each.

KEY WORDS: ethics; innovation; practice variation; decision making; decision analysis; care giving.

J Gen Intern Med 23(Suppl 1):27-31

DOI: $10.1007 / \mathrm{s} 11606-007-0410-2$

(C) Society of General Internal Medicine 2007

\section{BACKGROUND}

Current, evidence-based medicine is undergoing unprecedented development. ${ }^{1,2}$ New diagnostic and therapeutic methods resulting from biomedical research are being introduced into practice at an increasing rate. These innovations are not without risk and should not be permitted at the expense of ethical values. ${ }^{3,4}$ The challenge presented by the range of innovations in the practice of medicine is to determine whether new tests are acceptably sensitive and specific and whether new medications and procedures are acceptably safe and effective. ${ }^{5}$

In Canada and many other countries, medical intervention on human subjects deemed as "experimental care" requires that a protocol be submitted to a REB. ${ }^{6}$ This entity is equivalent to those in many countries, such as the Institutional Review Boards (IRB) in the USA, whose common mission is the protection of research subjects. This level of control no longer holds for procedures past the experimental stage. Thus, "innovative care" that uses a clinical-practice procedure that is beyond the experimental stage is not subject to review and monitoring by a REB. Nonetheless, without a commonly accepted definition of "innovation", the question persists as to what should qualify in practice as experimental care.

The research literature and certain national policies on research involving humans have examined definitions of experimental care, innovative care, and standard care. A procedure is classified under the concept of experimental care, ${ }^{7,8}$ if it involves an action whose clinical effectiveness has not been recognized and it is not yet known whether it will produce the benefits sought.

The concept of innovative care has been linked to several definitions viewing it both as a dynamic and complex process. Thus, its nature and degree can vary from 1 procedure to another. ${ }^{9,7}$ Still, other definitions place it on the continuum between research and practice. ${ }^{2,10,11}$ In Quebec, ${ }^{12}$ innovative care sits astride newly accepted and experimental procedures: their efficacy has been demonstrated, but, for lack of experience, methods of application and precise indications for use remain to be specified. This definition emerges from Article 21 of the Civil Code of Québec, according to which "Care considered by the ethics committee to be innovative care required by the state of health of the person concerned does not constitute an experiment". The lack of precision of this language has been criticized. ${ }^{13}$ The less controversial concept 
of standard or commonly accepted care is applied to clinical procedures whose clinical effectiveness, indications for use, and protocols are well established.

The gray zone that divides experimental care in humans from innovative care within clinical practice can give rise to difficulties for Research Ethics Boards and health professionals called upon to distinguish one from the other; the more so as biomedical research and the practice of medicine often take place within a single institution, heightening the possibility of confusion as to a procedure's purpose. ${ }^{14}$

Such confusion can result in variations among the opinions of those responsible for classifying procedures. Classifying experimental care as innovative, allows for administering experimental procedures to patients without observing the ethical and scientific norms for experiments on humans, whereas classifying innovative care as experimental might subject it to excessive ethical and scientific monitoring and could considerably delay patients' benefiting from clinical advances.

These situations raise the questions: "At what point should clinical applications be subject to the same standards for protecting subjects as those required in research?" At what point does modification of a standard technique constitute more than a modification and become research? There is very little research that has studied the extent of variation in opinion between health professionals or REBs. ${ }^{15,16}$ This pilot study was designed therefore to investigate the extent and nature of this variation. clinical biochemists (CBs) were specifically chosen as the target health professional group for this study as they are responsible for determining whether a new diagnostic test should be introduced into the clinical repertoire. They may have medical or scientific training. This role has equivalence in many countries including that of a laboratory medicine specialist in the USA.

\section{OBJECTIVE}

This pilot study examines the way in which REBs (Institutional Review Boards) and CBs (laboratory medicine specialists) differ in their interpretation of what is research and what should be considered common practice versus innovation versus experimentation when introducing new procedures with patients. It also explores whether these groups agree on who is responsible for the ethical review of new procedures.

\section{Methods}

Demonstrating perceptible variation in criteria used to determine the status of a new practice presumes assessment of the same project. To this end, a case scenario "Evaluation of the Impact of Introducing BNP Analysis into Clinical Practice to Diagnose Heart Failure" was devised. The case scenario was based on a new practice of current interest and with considerable variation between centers on policies of adoption. ${ }^{17,18,19}$ It relates specifically to the development of a protocol for the introduction of a diagnostic test aimed at enhancing clinical effectiveness.

The test described in the case scenario (brain natriuretic peptide or BNP) has been approved for clinical use by the FDA. Its role in heart failure diagnosis has been shown to be very promising but this has still to be fully established. ${ }^{20,21}$ The case scenario identifies 4 objectives: determine the analytical performance in the local laboratory; determine guidelines for optimal use; correlate the BNP test with other measures of heart failure; and to evaluate the cost-benefit ratio. The test would be made available without any change required in normal practice. A comparison of practice data before and after test introduction would be carried out to evaluate impact on choice of other investigations; choice of treatment; length of hospital stay; and overall costs. This case scenario was developed to allow for targeting significant variation among decision-makers classifying a procedure at the interface between research on humans and clinical practice.

Three professional CBs and 2 REB members validated the case scenario to ensure its credibility and realism. A selfadministered questionnaire consisting of 6 questions was devised to gather participants evaluation regarding: (Question 1) whether or not the case scenario project constituted a research project (yes or no); (Guestion 3) how the procedure (test) described in the case scenario should be classified (for example, experimental, innovative, commonly accepted, or other); (Question 5) whether or not they thought the project required approval by a REB (yes or no); and if not, (Question 6) who should be responsible for project review. Answers to the closed questions $(1,3,5$, and 6$)$ in the original questionnaire are presented in Table 1. Open-ended questions 2 and 4 gave access to the different explanations given for questions 1 and 3. The Fisher's exact test was used to assess if the answers differed between CBs and REBs.

\section{Participants}

The case scenario and questionnaire were transmitted by post mail between May 2003 and February 2004 to 53 biomedical REBs across Canada highly concerned by ethical issues of clinical innovation in their institution and that had agreed to take part in the study. The biomedical REBs were explicitly

Table 1. Comparison of Responses of CBs and REBs to the 4 Closed Questions

\begin{tabular}{lccc}
\hline \hline & CBs & REBs & P value \\
\hline Number of participants & 37 & 27 & \\
Question 1: In your view, does this & & & .0160 \\
project constitute a research & & & \\
project? & $30(81 \%)$ & $14(52 \%)$ & \\
Yes & $7(19 \%)$ & $13(48 \%)$ & .0379 \\
No & & & \\
Question 3: In your view, how & & & \\
should one describe the new & & & \\
procedure that is the subject of & & & \\
this project? & $3(8 \%)$ & $2(7 \%)$ & \\
Experimental & $25(68 \%)$ & $9(33 \%)$ & \\
Innovative & $4(11 \%)$ & $7(27 \%)$ & \\
Commonly accepted & $5(14 \%)$ & $9(33 \%)$ & \\
Other & & & \\
Questions 5 and 6: In your view, & & & \\
does this project require approval & & & \\
by a REB? If not, who should be & & & \\
responsible for project review? & & & \\
Yes & $20(54 \%)$ & $12(44 \%)$ & \\
No & $17(46 \%)$ & $15(56 \%)$ & \\
Clinical Ethics Board & $2(5 \%)$ & $2(7 \%)$ & \\
Other authority & $8(22 \%)$ & $11(41 \%)$ & \\
Investigators themselves & $7(19 \%)$ & $2(7 \%)$ & \\
\hline
\end{tabular}


asked to handle this case scenario just like any other that might normally come to their attention in the course of their work and give it no special treatment. A further requirement was that the ethical review handed down should represent the review of the REB as a whole and not that of a single member speaking as an individual. Responses were accepted until April 2004.

During this period, the same case scenario and questionnaire were also transmitted by e-mail to members of the Canadian Society of Clinical Chemistry (CSCC), the Association des médecins biochimistes du Québec (AMBQ), and the members of the Société québécoise de biochimie clinique (SQBC).

Responses to the closed questions were analyzed quantitatively as percentages, presented in this study in table format. Analysis of the open-ended questions was by mixed classification using criteria identified in the scientific literature or derived from the data themselves. ${ }^{22,23}$

This project was approved by the REB of the Sherbrooke University Hospital Center (Centre hospitalier universitaire de Sherbrooke), in Sherbrooke, Quebec, Canada.

\section{RESULTS}

Twenty-seven biomedical REBs and 37 CBs in Canada participated in this pilot study. Questionnaires were transmitted to CB members of the Canadian Societies of Clinical Chemistry $(n=273)$ and to 53 Canadian biomedical REBs that had agreed to take part in the study. Fourteen percent of CBs and $51 \%$ of REBs participated. Table 1 presents the comparison of responses of CBs and REBs to the 4 closed questions.

Out of the participating CBs, $30(81 \%)$ deemed the case scenario to constitute research compared to $52 \%$ of REBs ( $p=$ .0160). Sixty-eight percent of the CBs considered the procedure as innovative compared to only $33 \%$ of the REBs. Only $8 \%$ of all participants (REBs and CBs) considered the procedure to be experimental, whereas $17 \%$ at the other end of the spectrum categorized it as being part of commonly accepted care. The REBs and CBs showed a considerable divergence of opinion with regard to the classification of the biochemical test procedure $(p=.0379)$.

There was no consensus among either the REBs or the CBs as to the authorities responsible for the ethical review of the case scenario. Of all 64 participants in the study (27 REBs and $37 \mathrm{CBs}$ ), $44 \%$ of REBs and $54 \%$ of CBs assigned this responsibility wholly or partly to the REB, however, the difference is not statistically significant ( $p=.6131)$.

The REBs tended to classify the case scenario as a research project and gave REBs ethical responsibility. CBs who designated REBs responsible did not necessarily view the case scenario as constituting a research project, however, some of them considered that REBs should oversee the introduction of the test procedure. Indeed, according to them, innovation associated with practice change may not be without risk.

\section{DISCUSSION}

This countrywide study brings to the forefront evidence of an underestimated and generally poorly understood challenge, namely, that of the ethical norms that need to be applied to the spectrum of innovation in clinical practice. At a time when the risks of health care are being widely announced, ${ }^{24,3}$ little attention is being paid to the different risks associated with the continual drive to import new diagnostics or other innovative procedures into clinical practice.

Both REBs and CBs were highly divided on whether the case scenario constituted research on humans. Divergence in this basic understanding could have important clinical impact with risks of inappropriate medical decisions, prolongation of institutional stay, and unneeded costs to the health system. All of these issues have ethical implications.

About half $(54 \%)$ of the CBs participating were likely to consider that such a clinical project should be brought before their REB before being implemented. The remainder would see no need to notify their REB, which would mean that the latter would be unaware that such a project was underway within their own institutions. Of the CBs, $27 \%$ would either consider other professional or hospital authorities responsible for ethical review, whereas 19\% would simply hold themselves responsible.

Among participating REBs, approximately half would view the project as research and thereby impose standard research practice on clinicians. This finding is of interest given that few respondent REBs $(7 \%)$ considered the test procedure experimental care. This gap could derive from the unclear distinction between research "on" humans and research "involving" humans and perhaps contribute to the fact that participating REBs did not agree on the limits of their sphere of competence. ${ }^{25}$ This is troubling, given the importance of the REBs' decision-making role in many jurisdictions. The remaining REBs, should they be consulted by clinicians, would refer them to other professional or hospital authorities (38\%) or not refer them at all (3\%). It may be supposed that participating REBs (27\%) and CBs (11\%) who classified the test procedure as commonly accepted care-a classification conferring exemption from ethical and scientific review by a REB-would be less likely, given their classification to consult colleagues or a REB.

Other than a clear tendency to identify the test procedure described in the case scenario as not being experimental care (93\%), no pronounced trend can be identified in the REBs' classification of the procedure. In contrast to the REBs, a majority of CBs (68\%) classified the test procedure as innovative care.

An effect of professional culture may account for the gap between the opinions of the 2 groups surveyed. REBs' criteria reveal the importance assigned to scientific, public, and widespread recognition. Conversely, CBs appear to view recognition of this kind as only one of the aspects to consider and give equal weight to criteria associated with clinical practice, which is considerably influenced by experience and context, such as other institutions' recognition of the test procedure and the professional and financial impacts of its implementation in their own institution.

The important variation between CBs and REBs in our pilot study addresses Agich's deeper question about the legitimate place of innovation in the clinical practice of medicine and the ethical standards that should apply. ${ }^{16}$ According to Agich, the complex processes characteristic of clinical innovation are often not reducible to a scientific protocol. They typically involve intuition, experience, and an evolving knowledge about the treatment and disease processes and the interaction between treatment and pathology. Whereas scientific knowledge 
and judgment are certainly involved, the formal methodology required in a scientific protocol is often not attainable or not useful in the early phases of development. He argues: "Given the widespread acceptance of a regulatory ethics paradigm (REP), bioethicists have not been inclined to examine closely the actual processes by which clinical innovations are developed and the initial conditions prerequisite for the application of the paradigm". Agich highlights this important omission because it has permitted the extension of a regulatory ethics paradigm into the domain of clinical innovation without an adequate assessment of the advantages and disadvantages of such an expansion. For now, innovation in clinical practice presents a moving target for the mandate of REBs.

It should be noted that REB participants in this survey may not be representative of the whole community of biomedical REBs in Canada. The findings we present in this study make no claims to predictive value or statistical validity. Their purpose is first and foremost to offer an analytical reflection of the decisions and decision-making criteria used by participating clinicians and REBs in reviewing a single clinical project.

\section{CONCLUSIONS}

This pilot study shows significant variation in opinion among participating REBs and CBs in determining whether a particular case scenario constitutes experimental, innovative, or commonly accepted practice. Among REBs, the divergence is especially noticeable. The odds of investigators getting 1 response or the other from a REB are roughly 50-50, effectively the same as a coin toss. This situation can only affect REBs' credibility for the worse.

Do these divergences entail a lower level of protection for subjects? It is not the purpose of our study to engage this question, but it ought to be addressed in the light of the decision-making practices of those responsible for interpreting and applying existing norms. Although it may be neither realistic nor desirable to seek highly reduced variation between CBs' and REBs' opinions, it is to be hoped that further criteria will be defined to reduce this variation to reasonable levels. This is all the more desirable given that, for a new test procedure to be deemed reasonable, peers of those introducing it must share that view of it. ${ }^{26}$ The variation in REB response suggests that there is an important need for further research and education to align ethics approval policies. The concern of the CBs regarding the scenario was that the test procedure described (in its current state of innovation) was still associated with uncertainty as to its potential benefit or harm. This was less evident to the REBs, which suggests that REBs may not be fully in touch with potential consequences or impact of innovations to patients. These findings are disturbing and suggest that this is an area where best practice policies need strengthening.

Innovation is a neglected area for ethics assessment. Further studies on a larger scale are necessary to review the concepts of experimental, innovative, and commonly accepted care. Our findings appear to confirm Agich's conclusion (2001): we will need to pay closer attention to the actual clinical, institutional, and professional processes that operate in the development of novel therapeutic interventions. The measures applied should also take account of the influence of professional culture, whether clinical or scientific, in the application of general definitions to a specific project.

Our pilot study looked at a particularly common type of innovation, namely, that of a new biochemical test. There is a need to further elaborate on the type of innovations in health care and categorize the nature of the risks associated with each. Further work could also look at why ethics committees might show this large extent of variation. Further research could have considerable implication for the education of both REBs and health care professionals. The concern of the latter is particularly interesting as it suggests that with appropriate education there would be a greater awareness amongst professionals of the risks linked to innovation and hence an improved culture of risk avoidance in an area that is likely to increase in complexity with biotechnological advance.

Acknowledgments: We wish to thank the Canadian Institutes of Health Research (CIHR), whose financial support made this pilot study possible. We also thank the participating REBs and CBs, and Diane Fafard, Denise Mathieu, Johane de Champlain, and Pierre Labossière for their support.

Potential Conflicts of Interest: None disclosed.

Corresponding Author: Johane Patenaude, PhD; Department of Surgery, Faculty of Medicine and Health Sciences, Université de Sherbrooke, 3001, 12th Avenue North, Sherbrooke, QC J1H 5N4, Canada (e-mail: Johane.Patenaude@USherbrooke.ca).

\section{REFERENCES}

1. Kerridge I, Lowe M, Henry D. Ethics and evidence based medicine BMJ. 1998;316(7138):1151-3.

2. Lind SE. Innovative medical therapies: Between practice and research. Clin Res. 1988;36:546-51.

3. Wilfond BS. Screening policy for cystic fibrosis: the role of evidence. Hastings Cent Rep. 1995;25:S21-3.

4. Tonelli MR. The philosophical limits of evidence-based medicine. Acad Med. 1998;73(12):1234-40.

5. Desjardins L. Le consentement à l'expérimentation clinique : Quelles sont les limites de l'acceptable et de l'inacceptable? Le Journal du Barreau. 1997;29(4). Available at: http://www.barreau.qc.ca/journal/ vol29/no4/consentement.html. Accessed December 18, 2006.

6. Canadian Institutes of Health Research, Natural Sciences and Engineering Research Council of Canada, Social Sciences and Humanities Research Council of Canada. Tri-council policy statement: ethical conduct for research involving humans. Ottawa: 1998. Updated June 2003.

7. Fost N. Ethical dilemmas in medical innovation and research: Distinguishing experimentation from practice. Semin Perinatol. 1998;22 (3):223-32.

8. Weisstub DN, Verdun-Jones S. Pour une distinction entre l'expérimentation thérapeutique et l'expérimentation non thérapeutique. Revue de Droit, Université de Sherbrooke. 1996-97;27:49-87.

9. Prentice ED, Gordon BG, Lin MH. Determining when a clinical activity should be classified as research requiring institutional review board review. J Extra-Corpor Technol. 1997;29(2):88-91.

10. American College of Physicians. Ethics manual. Fourth edition. American College of Physicians. Ann Intern Med. 1998;128:576-94.

11. National Institute of Health. Institutional Review Board for studies involving human participants at the National Institute of Environmental Health Sciences. Available at: http://www.niehs.nih.gov/about/orgstructure/boards/irb/terms.cfm. Accessed August 29, 2007.

12. Conseil d'évaluation des technologies de la santé. La transplantation au Québec, rapport préliminaire sur l'efficacité, les coûts et les caractéristiques organisationnelles, Montréal 1991;2-3. 
13. Kouri RP, Philips-Nootens S. L'expérimentation et les soins innovateurs : l'article 21 C.c.Q. et les affres de l'imprécision. Revue de Droit, Université de Sherbrooke. R.D.U.S. 1996-1997;27:89-137, p.101.

14. Edgar H, Rothman DJ. The institutional review board and beyond Future challenges to the ethics of human experimentation. Milbank $Q$ 1995;73(4):489-506.

15. Moutel G, Leroux N, Hervé C. Analysis of a survey of 36 French research committees on intracytoplasmic sperm injection. Lancet. 1998;351(9109):1121-3.

16. Agich GJ. Ethics and innovation in medicine. $J$ Med Ethics 2001;27:295-6.

17. Hearnshaw $\mathbf{H}$. Comparison of requirements of research ethics committees in 11 European countries for a non-invasive interventional study. BMJ. 2004;328:140-1

18. Silverman H, Hull SC, Sugarman J. Variability among institutional review boards' decisions within the context of a multicenter trial. Crit Care Med. 2001;29(2):235-41.

19. Shah S, Whittle A, Wilfond B, Gensler G, Wendler D. How do institutional review boards apply the federal risk and benefit standards for pediatric research? JAMA. 2004;291:476-82.
20. Valli N, Georges A, Corcuff JB, Barat JL, Bordenave L. Assessment of brain natriuretic peptide in patients with suspected heart failure: Comparison with radionuclide ventriculography data. Clin Chim Acta. 2001;306(1-2):19-26.

21. Maron BJ, Tholakanahalli VN, Zenovich AG, et al. Usefulness of Btype natriuretic peptide assay in the assessment of symptomatic state in hypertrophic cardiomyopathy. Circulation. 2004;109:984-9.

22. Tashakkori A, Teddlie C. Handbook of Mixed-Methods In Social \& Behavioral Research. Thousand Oaks, CA: Sage; 2003. See Creswell, chap.8, pp. 209-40.

23. Gubrium JF, Holstein JA. The New Language of Qualitative Method. New-York: Oxford University Press; 1997.

24. World Medical Association. World Medical Association Declaration of Helsinki: ethical principles for medical research involving human subjects. JAMA. 2000;284:3043-5.

25. McDonald M, Dickens B, Knoppers BM, et al. The Governance of Health Research Involving Human Subjects (HRIHS). Law Commission of Canada. 2000.

26. Wheeler R. One person's innovation is another's experiment. BMJ. 2000;320:1548. 